\title{
Les disparités régionales dans la Hongrie en transition
}

Regional disparities in the Hungarian transition

Regionale Disparitäten im Ungarn des Übergangs

József Nemes-Nagy

Traducteur : Michel Deshaies et Simon Edelblutte

\section{(2) OpenEdition}

\section{Journals}

Édition électronique

URL : http://journals.openedition.org/rge/2466

DOI : $10.4000 /$ rge. 2466

ISSN : 2108-6478

Éditeur

Association des géographes de l'Est

Édition imprimée

Date de publication : 1 janvier 2003

ISSN : 0035-3213

\section{Référence électronique}

József Nemes-Nagy, "Les disparités régionales dans la Hongrie en transition », Revue Géographique de l'Est [En ligne], vol. 43 / 1-2 | 2003, mis en ligne le 07 décembre 2010, consulté le 08 septembre 2020. URL : http://journals.openedition.org/rge/2466 ; DOI : https://doi.org/10.4000/rge.2466

Ce document a été généré automatiquement le 8 septembre 2020

Tous droits réservés 


\section{Les disparités régionales dans la Hongrie en transition}

Regional disparities in the Hungarian transition

Regionale Disparitäten im Ungarn des Übergangs

József Nemes-Nagy

Traduction: Michel Deshaies et Simon Edelblutte

\section{NOTE DE L'ÉDITEUR}

Cet article a été traduit de l'anglais, adapté et mis en forme par Michel Deshaies et Simon Edelblutte (CERPA / Université Nancy 2).

\section{NOTE DE L'AUTEUR}

Cette recherche a été soutenue par le projet PHARE P95-2024R intitulé « Politiques régionales de développement économique ».

\section{Introduction}

Les changements politiques survenus en 1989-90 ont provoqué un bouleversement de la structure économique et sociale de la Hongrie et, en conséquence, une profonde modification de l'organisation régionale héritée des périodes précédentes. Ces mutations affectent chaque région, chaque partie du pays - en ce sens, la mutation est globale et de grande ampleur - mais introduisent néanmoins de fortes disparités spatiales. Ainsi, et alors que dans certaines régions, la transition conduit à des situations critiques, ailleurs, elle s'effectue dans des conditions relativement équilibrées; dans certains cas, elle a même des effets positifs qui, à court terme, finissent par l'emporter sur les aspects négatifs. La Hongrie illustre donc bien 
l'excellente terminologie de Gorzelak (1996) sur les évolutions régionales durant les périodes de transition. Quatre types d'évolution sont distingués, en fonction de la situation avant la transition: discontinuité positive, discontinuité négative, continuité positive ou continuité négative (tableau 1).

Tableau 1 : Typologie des dynamiques régionales durant la transition

\begin{tabular}{|c|c|c|c|c|}
\hline & & \multicolumn{3}{|c|}{ Evolution dans les années 1990} \\
\hline & & Dynamisme & Stagnation & Déclin \\
\hline \multirow{3}{*}{$\begin{array}{l}\text { Niveau } \\
\text { de développement } \\
\text { à la fin de } \\
\text { la période communiste }\end{array}$} & $\begin{array}{l}\text { Au-dessus } \\
\text { de la moyenne }\end{array}$ & $\begin{array}{l}\text { CONTINUITE POSITIVE } \\
\text { Budapest } \\
\text { et son agglomération }\end{array}$ & & \\
\hline & Moyenne & $\begin{array}{l}\text { DISCONTINUITE } \\
\text { POSITIVE } \\
\text { Nord-Ouest } \\
\text { et grandes villes }\end{array}$ & $\begin{array}{l}\text { Sud-Ouest } \\
\text { et villes moyennes }\end{array}$ & $\begin{array}{l}\text { DISCONTINUITE } \\
\text { NEGATIVE } \\
\text { Nord }\end{array}$ \\
\hline & $\begin{array}{l}\text { En dessous } \\
\text { de la moyenne }\end{array}$ & & $\begin{array}{l}\text { Sud-Est } \\
\text { et petites villes }\end{array}$ & $\begin{array}{c}\text { CONTINUITE } \\
\text { NEGATIVE } \\
\text { Nord-Est et villages }\end{array}$ \\
\hline
\end{tabular}

source : Gorzelak (1996)

2 Des années 1960 jusqu'au milieu des années 1980, les différences de développement et de richesse entre les différentes villes et les différentes régions sont restées relativement stables (Nemes-Nagy, 1990a). Cependant, dès le début des années 1980, la mise en place par le régime socialiste d'une économie de marché restreinte a permis la floraison de petites entreprises destinées à desservir les marchés urbains (Nemes-Nagy, Ruttkay, 1987 ; Sik, 1994). Elles étaient concentrées surtout dans la capitale, dans les grandes villes et dans la partie ouest du pays; ces régions ont donc commencé à prendre l'avantage sur les autres. Cela a provoqué des mutations sociales et économiques à l'origine d'une redistribution spatiale qui s'est accélérée après 1989, avec le développement de l'économie de marché (Cséfalvay, Nikodémus, 1991; Cséfalvay, 1995). Plus tard, durant les années 1990, c'est l'inégale répartition des investissements étrangers qui a accentué encore les disparités régionales (Van Hastenberg, 1996 ; Enyedi, 1996b).

3 Actuellement, les inégalités régionales en Hongrie se déclinent selon trois échelles se recoupant plus ou moins (Cséfalvay, 1993 ; Rechnitzer, 1993a-b ; Nemes-Nagy, 1995ad ; Enyedi, 1996a) : l'échelle nationale avec le dualisme entre Budapest et le reste du pays, l'échelle inter-régionale avec les inégalités entre l'est et l'ouest et enfin l'échelle intra-régionale, avec les inégalités villes/campagnes. La combinaison entre ces trois échelles est visible sur la figure 1. Ces inégalités montrent une grande stabilité depuis le début de la transition. En effet, à la fin des années 1990, la situation inter- et intra-régionale reste fondamentalement comparable à ce qu'elle était au début de la décennie et les évolutions essentielles, amorcées dans la seconde moitié des années 1980 et la première moitié des années 1990, sont donc déterminantes pour l'évolution future des inégalités régionales. Ces trois échelles, ainsi que les perspectives d'évolution des disparités régionales, constitueront la trame de cet article. 
Figure 1 : Disparités spatiales à différentes échelles en Hongrie à la fin du XXe siècle

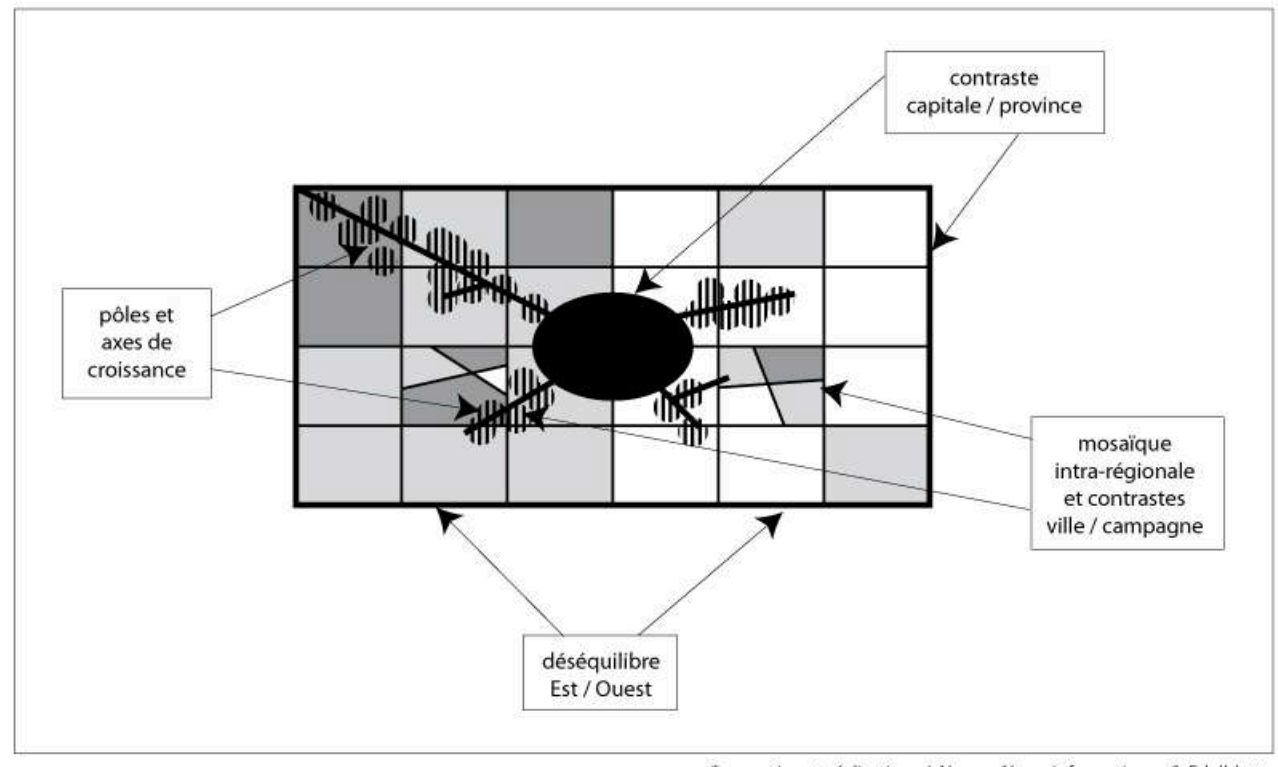

Conception et réalisation : J. Nemes-Nagy, informatique : S. Edelblutte

\section{Les inégalités inter-régionales}

4 À l'origine des inégalités inter-régionales, plusieurs facteurs peuvent être distingués; ils sont classés en fonction de l'époque durant laquelle ils ont commencé à jouer un rôle et en fonction de leur persistance :

- des facteurs de longue durée et d'une grande pérennité, intégrant la dimension historique, les conditions naturelles, les caractéristiques psycho-sociologiques de la population et le réseau urbain ;

- des facteurs à moyen terme comme les réorganisations régionales de la période socialiste ;

- des changements récents ayant des effets à court terme et notamment le changement de système politique et les conséquences d'une évolution vers l'économie de marché.

\section{A. La prédominance de Budapest}

5 À l'échelle européenne, la caractéristique principale de la Hongrie est sa macrocéphalie urbaine. La prééminence de la capitale dans les domaines économiques, culturels, intellectuels, politiques, lui assure finalement un pouvoir hors de proportion avec son poids démographique, pourtant écrasant - environ $20 \%$ de celle du pays (Barta, Conti, 1994).

6 En bref, on peut dire que le pays est constitué d'une grande ville et de sa périphérie. Les origines historiques $\mathrm{du}$ dualisme entre la capitale et le pays remontent approximativement à environ un siècle. Cependant, les différences de développement économique entre la capitale et le reste du pays se sont accrues depuis une décennie, notamment à partir des années 1980 et du boom des petites entreprises socialistes. Les décennies précédentes avaient par contre été marquées par l'importance croissante donnée au reste du pays en raison de la décentralisation industrielle et du développement constant de l'agriculture. 
7 La capitale et son agglomération représentent donc l'aire de dynamisme la plus importante en Hongrie (Lengyel, 1993; Probáld, 1995; Szirmai, 1998; Barta, Keresztély, 1999). Dans chaque secteur économique, la capitale s'élève nettement audessus du reste du pays. Le produit national brut par habitant y est d'environ $80 \%$ supérieur à la moyenne nationale (Kovács, 1993), bien que le niveau de développement économique n'y soit que de la moitié de la moyenne de l'Europe des 15. Le dualisme entre la capitale et la province apparaît de la manière la plus directe à travers la sélection des branches les plus dynamiques de l'économie.

8 Cette tendance se poursuit parallèlement à un processus de nivellement par le bas du reste du pays. Les régions considérées autrefois comme les plus développées (en particulier l'axe industriel sud-ouest/nord-est) ont subi une crise structurelle et sont aujourd'hui largement économiquement déprimées. Leur avantage initial sur les autres régions a diminué (tableau 2).

Tableau 2 : Développement économique et disparités régionales

\begin{tabular}{|c|c|c|c|c|c|c|}
\hline \multirow{2}{*}{ 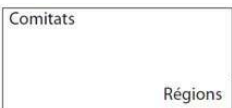 } & \multicolumn{3}{|c|}{$\begin{array}{c}\text { PNB par habitant } \\
(\text { Hongrie }=\text { indice 100) }\end{array}$} & \multicolumn{3}{|c|}{ Evolution en \% } \\
\hline & 1975 & 1994 & 2000 & 1975-1994 & $1994-2000$ & $1975-2000$ \\
\hline Budapest & 139 & 180 & 195 & 41 & 15 & 56 \\
\hline & 61 & 76 & 78 & 15 & 2 & 17 \\
\hline Hongrie Centrale & 114 & 146 & 152 & 32 & 6 & 38 \\
\hline Fejér & 106 & 96 & 127 & -10 & 31 & 21 \\
\hline Komárom-Esztergom & 131 & 80 & 83 & -51 & 3 & -48 \\
\hline Veszprém & 116 & 80 & 85 & -36 & 5 & -31 \\
\hline Transdanubie Centrale & 117 & 86 & 100 & -31 & 14 & -17 \\
\hline Gyir-Moson-Sopron & 111 & 103 & 134 & -8 & 31 & 23 \\
\hline Vas & 82 & 103 & 114 & 21 & 11 & 32 \\
\hline Zala & 88 & 94 & 85 & 6 & -9 & 3 \\
\hline Transdanubie Occidentale & 96 & 101 & 114 & 5 & 13 & 18 \\
\hline Baranya & 108 & 84 & 76 & -24 & -8 & -32 \\
\hline Somogy & 71 & 76 & 68 & 5 & -8 & -3 \\
\hline Tolna & 77 & 94 & 83 & 17 & -11 & 6 \\
\hline Transdanubie du Sud & 88 & 84 & 75 & -4 & -9 & -13 \\
\hline Borsod-Abaúj-Zemplén & 111 & 70 & 65 & -41 & -5 & -46 \\
\hline Heves & 100 & 73 & 71 & -27 & -2 & -29 \\
\hline Nógrád & 77 & 62 & 54 & -15 & -8 & -23 \\
\hline Hongrie du Nord & 102 & 70 & 65 & -32 & -5 & -37 \\
\hline Hajdú-Bihar & 83 & 83 & 71 & 0 & -12 & -12 \\
\hline Jász-Nagykun-Szolnok & 93 & 79 & 67 & -14 & -12 & -26 \\
\hline Szabolcs-Szatmár-Bereg & 59 & 62 & 54 & 3 & -8 & -5 \\
\hline Grande Plaine du Nord & 77 & 74 & 63 & -3 & -11 & -14 \\
\hline Bács-Kiskun & 79 & 77 & 68 & -2 & -9 & -11 \\
\hline Békés & 89 & 80 & 66 & -9 & -14 & -23 \\
\hline Csongrád & 109 & 94 & 83 & -15 & -11 & -26 \\
\hline Grande Plaine du Sud & 91 & 83 & 72 & -8 & -11 & -19 \\
\hline $\begin{array}{l}\text { Rapport entre le comitat } \\
\text { le plus pauvre et le comitat } \\
\text { le plus riche }\end{array}$ & 2,36 & 2,90 & 3,61 & & & \\
\hline Mème rapport sans Budapest & & 2,22 & 1,66 & & & \\
\hline
\end{tabular}

Sources : 1975 : estimations de J. Nemes-Nagy / 1994 et 2000 : CSO

\section{B. Le contraste ouest-est}

9 Les différents indices économiques laissent apparaître un contraste ouest-est se différenciant de la traditionnelle division nord-sud. Le produit national brut par habitant (PNB/hab) de toutes les régions de l'ouest atteint au moins $80 \%$ de la moyenne nationale. Au contraire, l'Est, mais à l'exception du comitat de Csongrád, est très en deçà de la moyenne de 1995. La région qui présente le plus fort dynamisme et relativement peu de signes de crise regroupe deux comitats situés le long de la frontière occidentale : les comitats de Vas et surtout de Gylr-Moson-Sopron. Par contre, 
le pôle négatif, à l'extrémité nord-est du territoire, est constitué par les comitats de Borsod-Abaúj-Zemplén, Nógrad et Szabolcs-Szatmár-Bereg. Il y a à peine 10 ans, c'est le comitat de Komárom-Esztergom qui venait en tête de liste et le comitat de Borsod-Abaúj-Zemplén appartenait aussi au groupe des régions les plus développées. Seul le comitat de Szabolcs-Szatmár-Bereg pouvait être considéré comme une région traditionnellement sous-développée.

$\mathrm{Du}$ point de vue de l'avantage que peut conférer la situation géographique, il faut souligner le rôle que jouent les déterminants de longue durée, certains remontant à plusieurs siècles. Ce ne sont pas seulement les contacts récents entre entreprises qui sont plus intenses le long de la frontière occidentale, mais aussi les contacts entre les personnes, tant en ce qui concerne les opportunités d'emploi que les achats ou les échanges d'informations. La longue histoire commune de l'Autriche et de la Hongrie, malgré la coupure de l'époque communiste, favorise ces liens. Par contre, dans les régions orientales, il n'y a pas de tradition de contact à un degré aussi fort. En conséquence, en plus du niveau de développement économique, les atouts des régions de l'ouest sont aussi d'ordre psycho-sociologique et comportent des éléments mentaux et culturels.

11 Le dynamisme actuel des régions de l'ouest repose donc en premier lieu sur leur position géographique favorable. Ces régions sont en effet les plus proches de la zone qui, en Europe, présente le dynamisme le plus fort, les Alpes, le sud de l'Allemagne et l'Italie du Nord. Un autre atout de la zone frontalière occidentale est que, pour des raisons politiques et militaires, cette région a été épargnée par le développement de l'industrie lourde à l'époque du régime socialiste. Aussi, lors du changement de régime politique, cette région présentait une structure économique plus moderne et surtout plus souple, plus capable d'évolution dans un contexte économique exigeant plus de compétences et de travail qualifié (Rechnitzer 1996; Dıry, 1997, Berényi, 2002). La région est d'ailleurs déjà très largement en relation quotidienne avec les régions autrichiennes voisines. Ces facteurs reposent ainsi sur les atouts psycho-sociologiques acquis au cours d'un passé ancien pendant lequel s'est forgé l'esprit d'entreprise et de management.

De tous les secteurs économiques, c'est l'industrie qui est la plus affectée par la restructuration régionale marquée par la substitution du dualisme est-ouest à la traditionnelle inégalité nord-sud (Papp, Tóth, 1991; Hrubi, 1993 ; Nemes-Nagy, 1997). C'est ce que démontrent clairement les rythmes de croissance de la production industrielle, très différents suivant les régions. Le principal changement régional résultant de ces évolutions est l'effondrement de l'axe industriel nord-est/sud-ouest qui avait semblé être, pendant les quarante dernières années, l'élément le plus stable du cadre spatio-économique hongrois. Les seules exceptions notables à cette évolution négative sont la ville de Székesfehérvár qui montre quelques signes de lent redressement ainsi que, un peu plus récemment, les environs de la ville de Tatabánya. La capitale, située au centre de cet axe, a également cessé d'être un centre industriel et est devenue un centre financier, commercial et politique, avec une structure et une organisation spatiale radicalement transformées.

13 La crise qui affecte les régions de l'est a plusieurs origines. La première cause n'est pas tant à rechercher dans des dispositions locales désavantageuses que dans un mécanisme spécifique caractéristique de la fin des années 1980 et de la première moitié des années 1990. En effet, pendant cette période, les firmes des régions développées (en particulier 
celles de la capitale) ont transféré leur crise vers les périphéries en licenciant les migrants pendulaires originaires de ces régions et en commençant à y fermer leurs petites filiales rurales (Csésfalvay, 1993b ; Schwertner, 1994 ; Dorenbos, 1996). Le second facteur pourrait être le fait que ces comitats du nord-est ont été les plus profondément affectées par l'effondrement des marchés de l'est (en premier lieu l'URSS) dans l'industrie lourde et la production agricole de masse. Le troisième élément important est le sous-développement des infrastructures inter-régionales qui a incité les investissements étrangers à rester dans la partie ouest du pays. Ainsi, dans l'est, les rares capitaux étrangers investis l'ont été uniquement dans les entreprises ayant les marchés les plus prometteurs.

14 Le gradient ouest-est s'observe non seulement au niveau inter-régional, mais aussi au niveau intra-régional et au niveau des éléments du réseau urbain.

\section{Les disparités intra-régionales}

15 La partie précédente a donné un aperçu des inégalités inter-régionales, mais il existe aussi des inégalités à l'intérieur des comitats (Nemes-Nagy, Ruttkay 1991). Les régions dynamiques du pays (la capitale et son agglomération, la partie ouest et certaines villes de province) ont expérimenté en premier ces inégalités liées aux potentialités internes variées de chacun de ces territoires.

\section{A. Facteurs d'inégalités et typologie des sous-régions}

16 Une grande partie du pays souffre de problèmes anciens ou plus récemment apparus qui font que de nombreux espaces sont des zones à problèmes dont la situation ne pourrait même pas être améliorée par la politique de développement régional. À l'échelle interrégionale, l'ouest plus stable (le nord-ouest en particulier) et les parties orientales en crise (le nord-est) se distinguent nettement les unes des autres. Par rapport à ces pôles périphériques, les ressources économiques et sociales mobilisées dans le centre du pays ont été tout juste suffisantes pour assurer une relative stabilité, mais pas assez cependant pour permettre un véritable développement. Cependant, à une échelle plus fine, de multiples facteurs provoquent des fortes différences intra-régionales :

- l'absence de ressources naturelles locales favorables, notamment pour l'agriculture ;

- la crise du secteur industriel, en particulier de l'industrie lourde très concentrée dans de petites régions ;

- l'archaïsme des infrastructures, la déficience des approvisionnements et des réseaux de communication entre les villes et les campagnes ;

- la situation frontalière (à l'exception de la frontière occidentale) ;

- la dégradation de l'environnement de certaines régions ;

- la crise démographique (vieillissement, dépopulation) dans les régions rurales.

Sur ces bases, on peut distinguer 8 catégories principales de petites régions (tableau 3), que l'on peut d'ailleurs pratiquement toutes retrouver dans le comitat de BorsodAbaúj-Zemplén, au nord du pays. D'autres situations, moins variées existent dans certains comitats. Celui de Szabolcs-Szatmár-Bereg, au nord-est, présente, à cause de son caractère agraire, de ses infrastructures archaïques et de sa situation frontalière, de très nombreuses petites régions sous-développées. Plusieurs autres comitats de la Grande Plaine sont d'ailleurs caractérisées par une situation similaire. 
Tableau 3 : Types de petites régions selon leur développement

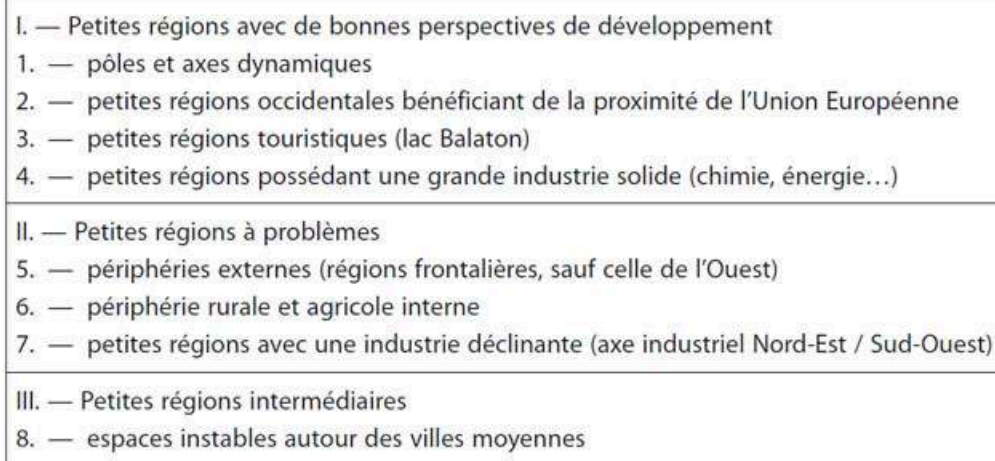

\section{B. Des inégalités à nuancer}

Dans l'Ouest, pourtant globalement en meilleure situation globale que l'Est, les régions rurales à petits villages de la Transdanubie méridionale présentent une situation instable à la fois démographiquement et économiquement. De même, le bassin industriel et minier du centre de la Transdanubie subit une crise similaire à celle que connaît le comitat de Borsod-Abaúj-Zemplén.

Un fait particulièrement typique est que, dans chaque comitat, la région qui entoure le centre administratif est dans une situation économique relativement meilleure: le chômage y est moins important, les nouvelles entreprises sont plus nombreuses et de nombreuses joint-ventures s'y sont développées. Les villes les plus importantes sont en effet attractives pour le commerce, regroupent les fonctions d'organisation de l'espace et attirent les nouvelles activités de services financiers et économiques. En combinaison avec les disparités régionales, la hiérarchie urbaine qui s'établit de la capitale jusqu'aux plus petits villages constitue un axe de différenciation sociale tout à fait primordial, à tel point que l'expression « gradient social urbain » n'est pas exagérée.

Plusieurs sous-régions affichent des progrès sensibles alors que, dans le même temps, elles présentent une multitude de signes d'aggravation de la crise. Ces régions instables, à l'avenir très incertain, se caractérisent par une économie duale et correspondent le plus souvent à des villes moyennes dynamiques et à leurs alentours en difficulté. Il y a donc encore une grande incertitude en ce qui concerne leur évolution qui peut être celle d'un développement rapide ou au contraire d'un profond déclin.

21 Tout comme les régions ayant une évolution positive, les régions à problèmes peuvent être classées en plusieurs catégories. Certaines se trouvent sur la rive droite du Danube, bien que les zones les plus vastes et les plus continues soient le plus souvent situées dans la partie orientale du pays.

22 La périphérie frontalière commence à la bordure sud de la Transdanubie ; il s'agit d'une région sous-développée, fondamentalement agraire, marquée par de hauts niveaux de chômage et qui a fort peu bénéficié des investissements étrangers. Au début des années 1990, la guerre, puis les tensions qui ont suivi entre la Croatie et la Yougoslavie, ont encore aggravé la situation de ces régions méridionales. Cette zone déprimée se 
prolonge ensuite vers le nord-est du pays où elle s'achève dans la région en crise de Szabolsc et Borsod.

Les régions déprimées ou menacées de crise sont situées le long d'un axe nord-est/sudouest (l'ancien axe industriel) jouxtant les régions évoquées précédemment. L'analyse régionale fait ressortir clairement que la notion de périphérie ne s'applique pas uniquement aux régions caractérisées par une situation géographique marginale, mais aussi à des régions à l'intérieur du pays (Nemes-Nagy 1996). Ces périphéries internes qui correspondent à des régions dont le centre est le plus souvent une petite ville, sont dans une situation similaire à celle des autres régions en crise (tableau 4) en raison de l'absence d'un centre urbain suffisamment attractif et de la crise de l'agriculture.

Tableau 4 : Dualités centre/périphérie dans la Hongrie en transition

\begin{tabular}{|l|c|c|c|}
\cline { 2 - 3 } \multicolumn{2}{c|}{} & \multicolumn{2}{c|}{ Localisation } \\
\cline { 2 - 4 } & \multirow{2}{*}{ Niveau de développement } & Centre & Périphérie \\
\cline { 2 - 4 } & Capitale & Frontière occidentale \\
\cline { 2 - 4 } & Périphérie & $\begin{array}{c}\text { Régions rurales du centre } \\
\text { (périphérie interne) }\end{array}$ & Autres zones frontalières \\
\hline
\end{tabular}

Un aspect particulièrement négatif de la nouvelle organisation régionale hongroise est le peu de connexions et de solidarité existant entre les unités régionales déterminées précédemment. Le pôle positif (la capitale et la partie ouest du pays) attire ainsi fortement la main-d'œuvre, utilise ses propres ressources et capte les investissements étrangers, mais ne participe que faiblement au développement du reste du pays. Il y a donc bien "attraction", mais non "diffusion", ce qui devrait renforcer, dans le futur, les inégalités régionales en Hongrie.

\section{L'évolution future des disparités régionales hongroises}

Les années à venir devraient être dominées par les forces de différenciation économique qui étaient déjà à l'œuvre au début des années 1990 (Nemes-Nagy, 1994). En référence au tableau 3, les inégalités entre les groupes de régions I et II devraient s'accroître, formant deux pôles opposant les régions dynamiques aux périphéries en difficulté.

\section{A. Les tendances globales}

Certains facteurs devraient exercer encore longtemps une influence sur les évolutions régionales (Enyedi 1994). C'est notamment le cas de la densité du réseau urbain. Au début de l'année 1998, 218 communes possédaient le statut légal de ville et regroupaient les deux tiers des habitants du pays. Cette population urbaine se caracté rise par un niveau croissant d'éducation et travaille surtout dans le secteur tertiaire ; elle est donc plus avancée dans une transition positive. Un autre facteur important pouvant exercer une influence sur les évolutions régionales est le nombre croissant de 
petites entreprises qui, pour la plupart, sont localisées dans les villes, mais ont tout de même une distribution spatiale plus uniforme que les grandes sociétés.

Un effet positif des processus décrits précédemment est que Budapest et la partie ouest du pays vont petit à petit devenir une région à l'économie moderne et tertiarisée. Par contre, les régions à problèmes conserveront un haut niveau de chômage et un accroissement de la polarisation interne au bénéfice des villes devrait se produire.

Malgré l'accroissement des différences, il est peu probable que se produisent des migrations de grande ampleur. Les facteurs démographiques n'influenceront donc pas beaucoup les différences de développement régional, d'autant plus que le développement des régions dynamiques est aujourd'hui plus lié à l'afflux important de capitaux et au développement d'innovations et moins aux disponibilités en maind'œuvre. L'exode rural avait d'ailleurs beaucoup diminué au cours des années 1980 et la relative jeunesse de la population des villes ne devrait pas le favoriser. Cependant, si la croissance économique se prolonge, Budapest pourrait probablement attirer de nouveau la main-d'œuvre des alentours.

Si le lancement de grands programmes de construction d'infrastructures, notamment routières, ne devrait générer qu'une migration temporaire de la main-d'œuvre, le développement global du réseau de transport peut permettre la migration des classes moyennes de la population urbaine vers les régions rurales environnantes bénéficiant d'un cadre naturel favorable. Ce processus pourrait stopper la dévalorisation des propriétés rurales les plus modernes.

\section{B. Les grandes phases du développement régional}

Dans l'hypothèse d'un développement économique continu, deux phases bien distinctes de développement régional devraient se succéder.

Durant la première phase, les principales régions affectées par le développement pourraient être les régions dynamiques du centre et de l'ouest, ainsi que les grandes villes. Pour le moment, ces régions concentrent les branches les plus dynamiques (banques, services), les agences de tourisme en expansion, etc. Elles ont reçu les investissements occidentaux les plus importants et les plus modernes. Ainsi, plusieurs sociétés multinationales ont implanté leurs usines d'automobiles en Hongrie occidentale. Dans ces régions, de nouvelles voies routières commencent à jouer le rôle d'axes de développement. Le démarrage économique de ces régions devrait exercer une influence très différenciée sur les régions périphériques. En effet, dans certains cas, l'influence sera négative puisque les migrants pendulaires originaires des périphéries, à faible niveau de qualification et non employables dans les nouvelles activités, seront licenciés en premier. À l'inverse, une conséquence positive pour les régions périphériques peut être une diminution du chômage si se développent dans les régions de l'ouest des activités employant une main-d'œuvre relativement peu qualifiée. Cette phase sera certainement caractérisée par une forte différenciation sociale et régionale.

Pour que les régions à problèmes connaissent une croissance et un développement, il faudrait que le taux de croissance du pays se maintienne à environ 3 à $5 \%$. Le taux de croissance du PNB est resté supérieur à $4 \%$ de 1997 à 1999 atteignant même un taux de $5,4 \%$ en 2001 , les conditions semblent donc être réunies pour permettre aux régions en 
difficulté de connaître également un décollage économique, ce qui devrait ralentir la tendance à la différenciation régionale.

Cette deuxième phase du développement régional se produira seulement si le secteur dominant qu'est l'agriculture dans ces régions connaît une croissance continue (SüliZakar, Baranyai, 1996), alors que pour l'instant il est en plein déclin. L'avenir des régions à problèmes dépend largement de la façon dont la situation économique et politique évoluera dans les anciens pays communistes voisins qui constituent les partenaires naturels de la Hongrie. Dans la partie orientale du pays, des signes d'une volonté de coopération économique apparaissent, avec par exemple la constitution de l'Euro-région des Carpathes (Süli-Zakar, 1997).

\section{Conclusion}

À plus long terme, la réduction des inégalités régionales dépendra aussi largement de la volonté politique du pouvoir central. Les chances de croissance des régions périphériques (Groen, Visser, 1993) reposeront essentiellement sur le développement d'un réseau d'infrastructures de communication efficace et de sa mise en relation avec le réseau principal et avec celui des pays voisins.

Le développement des régions périphériques passe donc par une meilleure coopération du pouvoir central hongrois avec les États voisins. Cette coopération fluctue cependant en fonction des pouvoirs politiques en place dans les grandes capitales de l'Europe de l'Est.

La volonté politique centrale est d'autant plus importante que, jusqu'ici et par rapport aux différents facteurs cités dans cet article, la politique d'aménagement du territoire joue un rôle bien moindre, même s'il n'est pas totalement négligeable (Nemes-Nagy, 1990b ; Horváth, 1993 ; OCDE, 1995 ; Csite, 1997 ; Bartke, 1997). La situation est d'autant plus mauvaise que les pouvoirs au niveau des comitats sont très limités en ce qui concerne la stimulation de l'activité économique. Les aides allouées au développement régional ne servent donc seulement qu'à atténuer les conséquences des crises locales.

Ainsi, la seconde phase de développement régional ne pourra commencer qu'avec des moyens plus importants et surtout une utilisation mieux coordonnée de ces fonds. À cet égard, l'adhésion à l'Union Européenne pourrait apporter des changements radicaux (Silince, 1987 ; Enyedi, 1996a ; Horváth, 1996, 1997 ; Horváth, Illés, 1997), notamment avec le projet de réorganisation du découpage régional aujourd'hui bien avancé1.

\section{BIBLIOGRAPHIE}

Barta Gy., Conti S. (1994). - Budapest's changing position in Europe and Hungary. In : Hajdú Z., Horváth Gy. - dir., European Challenges and Hungarian responses in Regional Policy, MTA RKK, Pécs, p. $170-182$. 
Barta Gy., Keresztély K. (1999). - Budapest, dynamisme et déséquilibres, Le Courrier des Pays de l'Est, $n^{\circ}$ 436, La documentation française, p. 35-50.

Bartke I. (1997). - Social driving forces and state regulation of regional economic development, Papers in Regional Science, $\mathrm{n}^{\circ}$ 2, p. 155-174.

Berényi I. (2002). - Die Auswirkungen der Privatisierung auf die Umgestaltung peripherer Räume in Ungarn, Europa Regional, n³/2002, p. 107-117.

Cséfalvay Z. (1993a). - Felharmadolt ország (Un pays en trois parties), Valóság, nº 7, p. 1-17.

Cséfalvay Z. (1993b). - Die Transition des Arbeitmarktes in Ungarn. Konsequenzen für sozialraümliche Entwicklung, Petermans Geographische Mitteilungen, ${ }^{\circ}$ 1, p. 33-44.

Cséfalvay Z. (1995). - Raum und Gesellschaft Ungarns in der Übergangsphase zur Marktwirtschaft. In : Mesburger P., Klinger A. - dir., Von Plan zum Markt. Eine Untersuchung am Beispiel Ungarns, Physica-Verlag, Heidelberg, p. 80-98.

Cséfalvay Z., Nikodémus A. (1991). - Két századvég Magyarországon (Deux fins de siècle en Hongrie), Tér és Társadalom, n 4/1991, p. 69-88.

Csite A. (1997). - Vidékfejlesztési megközelítések Magyarországon 1970-1996 : az új regionális politika (La nouvelle politique régionale et rurale en Hongrie), Szociológiaia Szemle, nº 1, p. 79-96.

Dorenbos R.J. (1996). - Labour mobility flows between 1988 and 1992 in Hungary and Poland. In : Workshop Transition Processes in Eastern Europe, ESR, La Haye, p. 137-162.

Dry T. (1997). - Szellemi potenciál és m"uszaki fejlesztés az Éskaznyugat-Dunántúlon (Recherche/dévelopement en Transdanubie du nord-ouest), Tér és Társadalom, n 1/1997, p. 57-72. Enyedi Gy. (1994). - Regional and urban development in Hungary until 2005. In : Hajdú Z., Horváth Gy. - éd, European Challenges and Hungarian Responses in Regional Policy, MTA RKK, Pécs, p. 239-253.

Enyedi Gy. (1996a). - Regionális folyamatok Magyarországon az átmenet idıszakában (Évolution régionale en Hongrie durant la transition), Hilscher Rezsı Szociálpolitikai Egyesület, Ember-településrégió sorozat, Budapest, $157 \mathrm{p}$.

Enyedi Gy. (1996b). - Külföldi müködıtıke befektetések hatása a regionális fejlıdésre Magyarországon (Les investissements directs étrangers et le développement régional en Hongrie). In : Dövényi Z. - éd, Tér-Gazdaság-Társadalom, MTA FKI, p. 247-256.

Gorzelak G. (1996). - The Regional Dimension of Transformation in Central Europe, Regional Policy and Development Series 10, London, Regional Studies Association and Jessica Kingsley Publishers.

Groen R., Visser A. (1993). - Development chances for Békés County, Univ. of Utrecht, MTA RKK, Utrecht-Békéscsaba, 125 p.

Horváth Gy. (1993). - Entrepreneurship and regional policy in Hungary. In : Hajdú Z. - éd, Hungary, CRS, Pécs, p. 263-277.

Horváth Gy. (1996). - A magyar regionális politika és az európai kihívások (La politique régionale hongroise face au défi européen), Vezetéstudomány, $\mathrm{n}^{\circ}$ 1, p. 17-29.

Horváth Gy. (1997). - Európai integráció, keleti bıvítés és a magyar regionális politika (L'intégration européenne, l'élargissement et la politique régionale hongroise), Tér és Társadalom, $n^{\circ} 3 / 1997$, p. 17-56.

Horváth Gy., Illés I. (1997). - Regionális fejlıdés és politika (Politiques de développement régional), Európai Tükör, n 16, ISM, Budapest, p. 25-34. 
Hrubi L. (1993). - Restructuring in a depressed zone and the role of new small enterprises : the case of Baranya county. In : Horváth Gy. - éd, Development strategies in the Alpine-Adriatic region, p. 183-207.

Kovács Cs. (1993). - A települési és térségi jövedelem egynlıtlenségek az adóköteles jövedelmek és a nyugdíjak alapján (Les inégalités spatiales en matière de revenu en Hongrie). In : Enyedi Gy. - éd, Társadalmi-területi egyenlitlenségek Magyarországon, KJK, Budapest, p. 359-383.

Lengyel I. (1993). - The Hungarian banking system in transition. In : Hajdú Z. - éd, Hungary, CRS, Pécs, p. 117-138. And In : Geojournal, Vol. 32, n 4, p. 381-391.

Nemes-Nagy J. (1990a). - Területi kiegyenlítıdés és differenciálódás Magyarországon (Convergences et divergences spatiales en Hongrie), Földrajzi Értesítı, p. 133-149.

Nemes-Nagy J. (1990b). - Regionális politika új feltételek között (Les nouvelles conditions de la politique régionale), Tér és Társadalom, n 1/1990, p. 89-103.

Nemes-Nagy J. (1994). - Regional disparities in Hungary during the period of transition to a market economy, Geojournal, Vol. 32, nº 4, p. 363-368.

Nemes-Nagy J. (1995a). — Soprontól Nyíradonyig - városok a piacgazdasági átmenetben (Les villes hongroises dans la transition économique), Comitatus, $\mathrm{n}^{\circ}$ 8-9, p. 15-22.

Nemes-Nagy J. (1995b). - A piacgazdasági átmenet terei (Les territories de la transition en Hongrie), Falu - Város - Régió, n 7-8, p. 6-11.

Nemes-Nagy J. (1995c). — A « gazdasági egészség » földrajzi képe Magyarországon (Géographie de la « santé économique » de la Hongrie), Földrajztanítás, n³-4, p. 4-11.

Nemes-Nagy J. (1995d). - Regional aspects of transition : development, problems and policies. Hungary, The Vienna Institute Monthly Report, $\mathrm{n}^{\circ}$ 1, p. 14-21.

Nemes-Nagy J. (1996). - Centrumok és perifériák a piacgazdasági átmenetben (Centres et périphéries dans la Hongrie en transition), Földrajzi Közlemények, p. 31-48.

Nemes-Nagy J. (1997). - Radikális változások a magyar ipar térszerkezetében. (Les changements radicaux dans la structure régionale de l'industrie hongroise), Földrajz - Hagyomány és Jövı, A 125 éves MFT jubileumi konferenciája, Budapest, $24 \mathrm{p}$.

Nemes-Nagy J., Ruttkay É. (1987). - A második gazdaság földrajza (Géographie de l'économie informelle), OTTGI, Budapest, $172 \mathrm{p}$.

Nemes-Nagy J., Ruttkay É. (1991). - Rural and urban forms of private enterprises in Hungary. In : Horváth Gy. - éd, Regional Policy and Local Government, CRS, Pécs, pp. 163-173.

OCDE (1995). - Social and labour market policies in Hungary, Paris, $241 \mathrm{p}$.

Papp J., Tóth J. (1991). - Ipari válságövezetek Magyarországon (Les territoires hongrois en déclin industriel), Közgazdasági Szemle, p. 778-797.

Probáld F. (1995). - Regionale Strukturen des Arbeitplatzangebotes in der Agglomeration von Budapest. In : Mesburger P., Klinger A. - dir, Von Plan zum Markt. Eine Untersuchung am Beispiel Ungarns, Physica-Verlag, Heidelberg, p. 182-208.

Rechnitzer J. (1993a). - Innovációs pontok és zónák, változási irányok a térszerkezetben (Centres, zones d'innovation et changements de l'organisation régionale). In : Enyedi Gy. - éd, Társadalmi-területi egyenlitlenségek Magyarországon, KJK, Budapest, p. 75-101.

Rechnitzer J. (1993b). - Szétszakadás vagy felzárkózás - A térszerkezetet alakitó innovációk (Cohésion, division et innovation dans le développement régional), RKK, Gyır, 165 p. 
Rechnitzer J. (1996). - Vas megye - sikeres modernizációs elmozdulás (Le comitat de Vas : une transition réussie). In : Csapó T. - éd, Átalakulás Vas megyében, MTA RKK -Vas Megyei Önkormányzat, Szombathely, p. 14-23.

Schwertner J. (1994). - Parázsló munkerıpiac (L'évolution du marché du travail), Tér és Társadalom, n 1-2/1994, p. 59-82.

Sik E. (1994). - From the multicolored to the black and white economy : the Hungarian second economy and the transformation, International Journal of Urban and Regional Research, Vol. 18, $\mathrm{n}^{\circ} 1$, p. 46-70.

Silince J.A. (1987). - Regional policy in Hungary : objectives and achievements, Transaction, Vol. 12 , p. $451-464$.

Süli-Zakar I. (1997). - A Kárpátok Eurorégió a régiók Európájában (L’Eurorégion des Carpates dans l'Europe des régions), Educatio, nº 3, p. 438-452.

Süli-Zakar I., Baranyai B. (1996). - A mezıgazdaság jövije, avagy a fenntartható fejlıdés esélyei az Alföldön (Agriculture et développement durable dans la Grande Plaine hongroise), Tér és Társadalom, nº 4, p. 125-137.

Szirmai V. (1998). - A budapesti régió társadalmi problémái (Les problèmes sociaux de la région de Budapest). In : Munkaeripiac és regionalitás, MKTA KK KI, Budapest, p. 187-196.

Van Hastenberg H. (1996). - Regional and sectorial characteristics of foreign direct investment in Hungary. In : Workshop on Transition Processes in Eastern Europe, ESR, La Haye, p. 121-136.

\section{NOTES}

1. Voir à ce propos l'article de Hajdú, dans ce numéro.

\section{RÉSUMÉS}

L'article traite des processus de transformation en Hongrie à différentes échelles régionales. L'accent est mis sur les relations entre la capitale et le reste du pays ainsi que sur les évolutions observables à l'échelle inter-régionale d'une part et intra-régionale d'autre part. La caractéristique essentielle des changements intervenus depuis les débuts de la transition est un accroissement des disparités régionales. Les éléments principaux des processus de transformation sont la tertiarisation rapide de l'économie à Budapest et l'augmentation des disparités est/ouest visibles dans l'évolution de plusieurs indicateurs socio-économiques (revenu moyen, chômage, investissements étrangers, etc.). L'auteur présente donc dans ce texte la nouvelle organisation régionale du pays et essaie d'en dégager les tendances futures majeures.

This paper analyses processes of transformation in Hungary at different regional levels. Emphasis is placed partly on relationships between the capital and the rest of the country, and also an observable movements at both the inter-regional and the intra-regional scale. The essential characteristic of changes since the beginning of the transition is an increase in regional disparities. The principal elements of these transformation processes are the rapid development 
of the tertiary economy in Budapest and the growth of East/West disparities, visible in the evolution of several socio-economic indicators (income, unemployment, foreign investment, etc.). The author summarises the new regional organisation of the country and attempts to identify major future trends.

Der Artikel behandelt Transformationsprozesse in Ungarn in verschiedenen regionalen Maßstäben. Der Akzent wird auf die Beziehungen zwischen der Hauptstadt und dem Rest des Landes gesetzt wie auch auf die sichtbaren Entwicklungen im interregionalen Maßstab einerseits, im intraregionalen andererseits. Das wesentliche Merkmal des Wandels seit Beginn des Übergangs ist ein Anwachsen der regionalen Disparitäten. Die wesentlichen Elemente des Transformationsprozesses sind die rasche Tertiärisierung der Wirtschaft in Budapest und die Zunahme der ost-westlichen Disparitäten, sichtbar in der Entwicklung mehrerer sozioökonomischer Indikatoren (mittleres Einkommen, Arbeitslosigkeit, Ausländische Investitionen usw). Der Autor stellt so in diesem Text die neue regionale Organisation des Landes vor und versucht dabei, die wesentlichen künftigen Tendenzen heraus zu stellen.

\section{INDEX}

Schlüsselwörter : Dualismus Ost-West, regionale Ungleichheiten, Regionalentwicklung, ungarischer Übergang

Keywords : East/West dualism, Hungarian transition, regional development, regional inequalities

Mots-clés : développement régional, dualisme est-ouest, inégalités régionales, transition hongroise

\section{AUTEURS}

\section{JÓZSEF NEMES-NAGY}

Department of Regional Geography - Eötvös Loránd University Budapest - 2, Ludovika tér, 1083 Budapest - HONGRIE 